\title{
Simple quadrature oscillator for BIST ${ }^{*}$
}

\author{
J. Raman, P. Rombouts and L. Weyten
}

\begin{abstract}
In this letter, a simple quadrature oscillator for built-in self-test (BIST) of integrated analog filters is proposed. A new hardware-efficient approach for amplitude control is described, with as main assets: (i) the technique requires little hardware which makes it very useful for BIST, (ii) the oscillation amplitude is well defined, and (iii) the distortion-level introduced by the amplitude control loop is under control of the designer.
\end{abstract}

Introduction Quadrature oscillators are used in a number of diverse applications. In telecommunication systems they are key building blocks in many (de)modulation schemes. However, quadrature oscillators are also useful in instrumentation and measurement systems, e.g., measuring an unknown impedance as a function of frequency [1]. The focus of this letter is on another instrumentation-related application: built-in self-test (BIST) of integrated analog filters. Here, the in-fase signal $v_{I}$ of the quadrature oscillator is applied to the analog filter to be tested (fig. 1). The output voltage of the filter is then demodulated by means of the quadrature signals for extracting amplitude and fase information of the analog filter transfer. In general, it is expected that the importance of such built-in self-test feature will steadily grow to guarantee proper operation of performance-critical analog blocks.

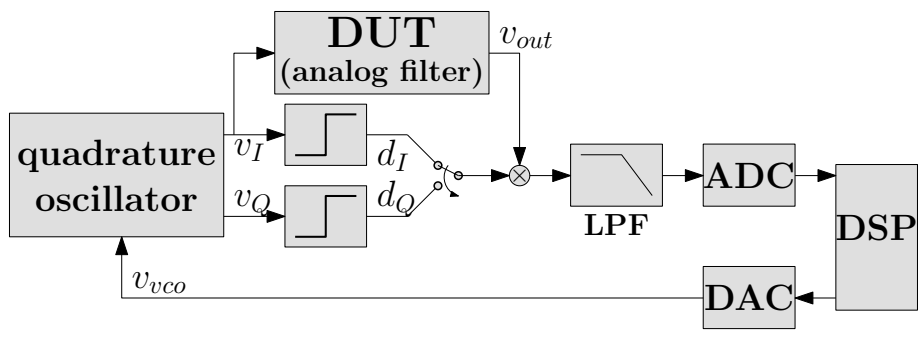

Figure 1: Example configuration for the built-in self-test of an analog integrated filter.

The requirements of quadrature oscillators strongly depend on the application. In telecommunication systems, typical

\footnotetext{
${ }^{*}$ This paper is a postprint of a paper submitted to and accepted for publication in IET Electronics Letters and is subject to Institution of Engineering and Technology Copyright. The copy of record is available at IET Digital Library.
}

oscillator requirements are a high frequency, a moderate tuning range, low phase noise, low quadrature phase errors, and often square-wave quadrature signals are satisfactory. In contrast, for instrumentation systems one requires most of the time two continuous-time sinusoidal signals, preferably with medium-to-low distortion. And while the required frequencies are typically lower for such applications, the needed tuning range is often much larger (e.g., one or two decades). These distinctive elements explain the often different approach followed.

For built-in self-test, there are important additional constraints. The extra test-circuits needed for BIST should be relatively small compared to the rest of the system. In this work, we will focus on the quadrature oscillator in fig. 1. It is assumed that with good circuit-reuse, integration of the rest of the test-setup can be done with a minimal amount of extra circuits: a DAC is reused for setting the frequency of the oscillator, and an ADC for converting the demodulated outputs to the digital domain. Further processing (e.g., extracting amplitude and phase) can be done in the digital domain. A lowpass filter should be present before the ADC to suppress unwanted demodulator frequencies. If not present already, a simple first-order filter could be added without significant additional cost. In order to simplify the demodulation process, the analog quadrature signals are first hard-limited. The initially needed analog multiplier then reduces to a chopper. The chopping operation becomes even trivial if the analog filter is implemented differentially, which is often the case.

Oscillator topology For the targeted application, the ability to sweep the frequency over at least one decade is mandatory. The need for a high tuning range favors the use of a two-integrator quadrature oscillator [2]. This well-known oscillator type is shown in fig. 2 (a). The real oscillator part consists of the feedback-loop containing the two integrators (grayed area). The two integrators are considered identical (except for unavoidable random mismatch), have differential outputs and are tunable. Because of the use of integrators with differential outputs, the sign inversion needed in the loop can be obtained trivially by cross-connecting. Using identical integrator circuits is beneficial to obtain a good quadrature relationship of the output signals, even in the pres- 


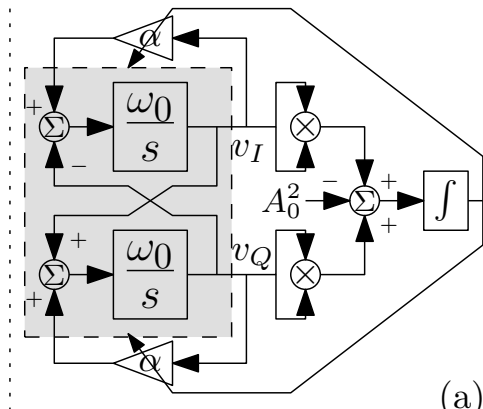

(a) :

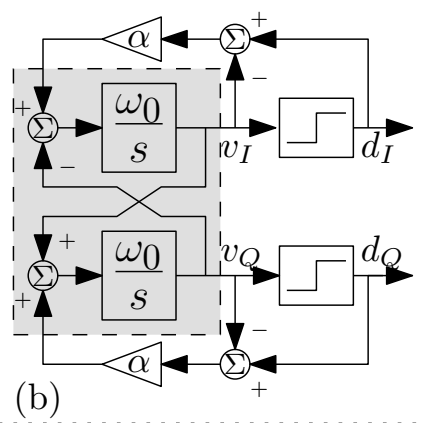

(c)

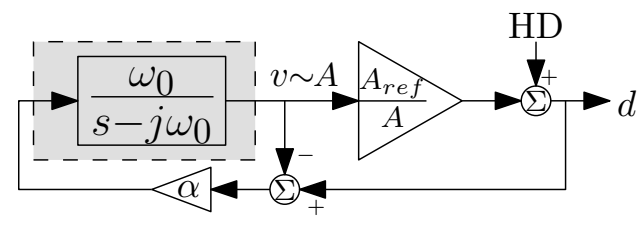

Figure 2: (a) Conventional two-integrator oscillator with amplitude control, (b) New oscillator with simplified amplitude control, (c) Representation as linearized system in the complex domain.

ence of parasitic effects such as additional poles or feedthrough signal paths. However, circuit imperfections do have the effect of pushing the poles away from the imaginary axis. For instance, the finite DC-gain of practical integrator circuits push the poles to the left-hand plane, which results in damping of the oscillator. As a result, the oscillator cannot function properly without some form of amplitude control. A traditional solution for this is also shown in fig. 2 (a). By squaring the quadrature signals and adding them up, we have a measure of the (squared) oscillation amplitude. Comparing this to a wanted reference level, we can setup a control loop to change the local feedback of the integrators. Obviously, the need for such an automatic gain control loop adds significantly to the complexity of the oscillator. Another approach for stabilizing the amplitude uses integrators with soft-limiting non-linearity [2]. While the complexity of this approach is low, the amount of non-linearity is very critical: over-compensation of damping results in severe distortion, while with under-compensation the circuit fails to oscillate. Even if one manages to operate in the intermediate region, the amplitude-level is not well controlled since it depends on a subtle balance of linear and nonlinear effects (with different temperature coefficients). Therefore, it is less appropriate for situations where the distortion of the outputs is important and needs to be guaranteed over a substantial temperature range. In the next section, an alternative control strategy that avoids these drawbacks is described.

Alternative amplitude control The new approach with respect to amplitude control is shown in fig. 2 (b). We already explained that for the built-in self-test of analog filters ac- cording to fig. 1, it is advantageous to have hard-limited versions of the quadrature signals available for phase-sensitive demodulation of the filter output. These hard-limited signals are also the basis of the amplitude control loop. Intuitively, the method can be understood as follows. The hardlimiting operation has the peculiar property that it converts an input sinusoidal signal with unknown amplitude $A$ into a signal with exactly the same frequency and phase, but now with a well-defined amplitude $A_{\text {ref }}=4 L / \pi$ determined by the output levels $\pm L$ of the limiter. We use this signal now as an implicit reference signal for setting up an amplitude control loop. First, we subtract the true oscillator output from it, which gives us a signal with a fundamental component proportional to $A_{r e f}-A$. This signal is fed back to the oscillator loop with a small gain $\alpha$ in such a way that the system behaves in a stable way. Because the loop gain is maximal at this fundamental frequency (theoretically infinite for ideal integrators), we expect that the systems will actually nullate the error $A_{\text {ref }}-A$, ultimately leading to a stable, well-controlled amplitude $A=A_{\text {ref }}$. In order to gain further insight into system properties (e.g., stability of the amplitude loop), we replace the hard-limiter by an amplitude-dependent gain, which is actually the describingfunctions approximation of this non-linearity. As explained in [3], it is advantageous to group quadrature-related signals into a single complex signal to simplify the system-level diagram: $v=v_{I}+j v_{Q}$ will represent the complex oscillator output, and $d=d_{I}+j d_{Q}$ the complex digital state of the hard-limiters. We then obtain the system-representation of fig. 2 (c). Note that we also introduced a term HD in this diagram, which represents the harmonic-distortion components introduced by the hard-limiter. From fig. 2 (c), the system pole $s=j \omega_{0}+\alpha(4 L / \pi A-1)$ is derived. As expected, only for $A=A_{\text {ref }}$ we obtain the purely imaginary pole $s=j \omega_{0}$, indicating oscillation at the frequency $\omega_{0} /(2 \pi)$. For $A<A_{r e f}$, the pole is unstable, resulting in an (exponential) increase of the amplitude. Likewise, for $A>A_{\text {ref }}$ the pole is in the left-hand plane and the oscillation amplitude will diminish. Hence, we obtain that $A=A_{r e f}$ is a stable operation point of the system, and (taking an appropriate time origin) the oscillator output can be described as $v(t)=A_{r e f} e^{j \omega_{0} t}$.

Distortion analysis The choice of $\alpha$ is influenced by two design considerations. First, $\alpha$ should be large enough to compensate the losses occurring in each integrator. On the other hand, the larger $\alpha$ becomes, the more harmonics created by the hard-limiters will couple to the oscillator, which affects the distortion of the quadrature output signals. In this section we derive an expression for the THD as a function of $\alpha$. Because the hard-limiter outputs which are simple square- 
wave functions, it is fairly easy to the derive the Fourierdecomposition:

$$
d=\frac{4 L}{\pi} \sum_{k \in \mathbb{Z}} \frac{e^{j(4 k+1) \omega_{0} t}}{4 k+1}
$$

Subtracting from this the expected fundamental frequency $A_{r e f} e^{j \omega_{0} t}$, we have an expression for HD in fig. 2 (c). The transfer from HD to the oscillator output (assuming the amplitude has settled to $\left.A=A_{r e f}\right)$ is given by $\alpha \omega_{0} /\left(s-j \omega_{0}\right)$. Combining these results, we can derive the following explicit expression for the (complex) amplitude of the $n$th harmonic:

$$
A_{n}= \begin{cases}\frac{\alpha}{n(n-1)} \frac{A_{\text {ref }}}{j} & n=4 k+1, k \in \mathbb{Z}_{0} \\ 0 & \text { others }\end{cases}
$$

Note that the only non-zero harmonics $|n|<7$ are $A_{-3}=$ $\alpha A_{r e f} / 12 j$ and $A_{+5}=\alpha A_{r e f} / 20 j$. These are the most important ones. From the expression for $A_{n}$, the total harmonic distortion is calculated to be $\operatorname{THD}(\mathrm{dB})=20 \log _{10}(\alpha / 10)$, independent of the oscillation amplitude. For practical values of $\alpha$ around 1/100, we obtain 60dB THD. Of course, in practical circuit implementations, also the distortion arising from integrator non-linearity needs to be evaluated, which can be substantial for $g_{m}-C$ integrators. Because integrator nonlinearity typically depends strongly on the oscillation amplitude, stabilizing this amplitude to an appropriate level allows to make actual designs with well-controlled THD.

Modeling accuracy The above results were derived with the linearized (describing function) model of fig. 2 (c). In addition to this, the authors have also conducted an exact analysis of the system, which is feasible but very cumbersome. It turns out that the exact oscillation condition requires that there exist real-valued solutions $a_{0}>0$ and $T>0$ of the following (transcendental) equation:

$$
a_{0}=\frac{\alpha(1+j) L}{\alpha-j} \frac{e^{(j-\alpha) \omega_{0} T / 4}-1}{e^{(j-\alpha) \omega_{0} T / 4}-j}
$$

This equation implies the following quarter-period relation of the oscillator output: $v(t)=j v(t-T / 4)$ (for the smallest $T$ solution). Together with the following exact solution for the first quarter-period:

$$
v(t)=e^{(j-\alpha) \omega_{0} t}\left[a_{0}+\frac{\alpha(1+j) L}{j-\alpha}\right]-\frac{\alpha(1+j) L}{j-\alpha}
$$

the oscillator output is completely determined analytically and is shown to be periodic with period $T$. By means of Taylor expansion, an approximate analytical solution for the oscillation frequency can be obtained as $\frac{1}{T}=$ $\frac{\omega_{0}}{2 \pi}\left(1-0.2146 \alpha^{2}\right)$. Consequently, the actual frequency is slightly lower than the expected frequency $\frac{\omega_{0}}{2 \pi}$, but this is only a second-order effect. Also for other results, deviations have been found to be of second order in $\alpha$. Since in practice, $\alpha$ will always be fairly small to limit the distortion, this validates the approximate analysis above.

Conclusion We have described a hardware-efficient way (essentially two hard-limiters) to stabilize the amplitude of a two-integrator quadrature oscillator. Based on the linearized model fig. 2 (c), we have established the effectiveness of the technique with respect to amplitude control, and derived an explicit expression for the harmonic distortion, which turns out to be suitable for practical built-in self-test.

Authors' affiliations: J. Raman, P. Rombouts and L. Weyten, Electronics and Information Systems Department, Ghent University, St. Pietersnieuwstraat 41, B-9000 Gent, Belgium

Please send electronic correspondence to: johan. raman @ elis.ugent.be

\section{References}

[1] B. Linares-Barranco, T. Serrano-Gotarredona, J. Ramos-Martos, J. Ceballos-Cceres, J. M. Mora, and A. Linares-Barranco, "A Precise $90^{\circ}$ Quadrature OTA-C VCO between 50-130 MHz," IEEE Int. Symp. on Circuits and Systems, ISCAS 2004, pp. 541-544

[2] L.B. Oliveira, J.R. Fernandes, I.M. Filanovsky, C.J.M. Verhoeven, M.M. Silva, "Analysis and Design of Quadrature Oscillators," Springer Science Business Media B.V., 2008.

[3] K. Martin, "Complex Signal Processing is not Complex,” IEEE Trans. Circuits Syst.-I, vol. 51, No. 9, pp. 1823-1836, Sept. 2004 